\title{
Integrating In-Situ and Ex-Situ Data Management Processes for Biodiversity Conservation
}

\author{
Karin R. Schwartz ${ }^{1 *}$, E. Christien M. Parsons ${ }^{1}$, Larry Rockwood ${ }^{2}$ and Thomas C. Wood ${ }^{3}$ \\ 'Department of Environmental Science and Policy, George Mason University, Fairfax, VA, United States, ${ }^{2}$ Department of \\ Biology, George Mason University, Fairfax, VA, United States, ${ }^{3}$ School of Integrative Studies, George Mason University, \\ Fairfax, VA, United States
}

There is an increasing need for a "one plan approach" for conservation strategies that integrate in-situ and ex-situ management processes. Zoological institutions contribute directly to threatened species conservation through paradigms, such as reintroduction, head-starting, supplementation, or rescue/rehabilitation/release. This in-situ/ex-situ integration necessitates collaboration at all levels of conservation action including planning, implementation, monitoring and assessment to drive adaptive management processes. Each component is dependent on the availability and accuracy of data for evidence to facilitate evaluation and adaptive management processes. The Zoological Information Management System (ZIMS), managed by Species360, is a centralized web-based information system used in zoological institutions worldwide to pool life history, behavior and health data and facilitate animal husbandry, health, and breeding management processes. Currently used for few integrated conservation programs, ZIMS is an innovative tool that offers a new opportunity to link data management processes for animals that spend a part of their lives under human care and part in their natural environment and has great potential for use in managed wild populations.

Keywords: one plan approach, conservation translocation, data management, species360, in-situ and ex-situ conservation

\section{INTRODUCTION}

The natural world is losing biodiversity due to the burgeoning human population that is perpetuating the unsustainable use of natural resources (Wilson, 2016). The overall goal of conservation is to mitigate the loss of biodiversity and preserve ecosystem services, species, and genetic diversity for the future. In this changing world, there is an increasing need for a "One Plan Approach" to develop multi-disciplinary conservation strategies that include the integration of in-situ (in the wild) and ex-situ (in zoological facilities) management processes when appropriate to do so (Byers et al., 2013). Zoological institutions may contribute to conservation directly through different conservation translocation programs including reintroduction, supplementation, head-start programs, or rescue-rehabilitation-release efforts. Integration of in-situ and ex-situ components of conservation programs necessitates collaboration at all levels of conservation action including planning, implementation, monitoring and assessment, to drive adaptive management processes. Conservation action is dependent on accurate, accessible data to guide the formation of action plans and carry out adaptive management processes. This requires increased integration of in-situ and ex-situ data management to provide the evidence-based science required for program assessment, planning, implementation, and adaptive management. 
In 2010, the Conference of the Parties (COP) for the 10th Convention on Biological Diversity (CBD) identified 20 new conservation targets (Aichi Targets) to form guidelines for biodiversity conservation through 2020 (Convention on Biological Diversity, 2010). Zoos and aquariums were noted as organizations that could contribute to "Strategic Goal C, Target 12 " i.e., "By 2020 the extinction of known threatened species has been prevented and their conservation status, particularly of those most in decline, has been improved and sustained." An assessment for the world zoo and aquarium community in 2013 identified conservation as a priority to increase contributions toward achieving Aichi Biodiversity Target 12 (Gusset et al., 2014).

In Decision VII/30 of the CBD, the COP agreed on a provisional list of global indicators to assess progress toward the 2010 targets (Convention on Biological Diversity, 2010). In a review of reports from $83 \%$ (159 of 193) of the Parties, only $36 \%$ included evidenced indicators backed up by data (Bubb et al., 2011). Only four evidenced relevant indicators for Aichi Target 12 emerged from the reports; 31 non-evidenced indicators also emerged, demonstrating that there were few data sets available, a lack of capacity for reporting on these indicators, or that these were low priorities. Thus, despite available modern digital technology, there was a paucity of data for assessment and implementation of conservation action.

Historically, conservation practices have been informed by ad hoc data and retrospective analysis based on anecdote and personal experiences (Pullin et al., 2004). Many early conservation translocation programs were afflicted by poor planning, lack of resources and release of inappropriate founders (confiscations, exotic pets or surplus animals from captive breeding programs) with limited or no post-release monitoring (Seddon et al., 2007). Beck et al. (1994) found a success rate of $11 \%$ when assessing 145 reintroduction projects involving captive animals between 1900 and 1991 (with the majority post 1970). Fischer and Lindenmayer (2000) reviewed 180 case studies and additional theoretical papers on animal translocations between 1980 and 2000 and found that only $26 \%$ of the programs were considered successful. The field of reintroduction biology has advanced since the reintroduction projects prior to 1990 (Ewen et al., 2012). The IUCN Reintroduction Specialist Group (RSG) developed the Guidelines for Reintroductions (IUCN/SSC, 1998) to improve the reintroduction processes. Use of the Reintroduction Guidelines improved pre-release planning, selection of founders, release protocols and postrelease monitoring processes and the overall success of these endangered species conservation programs (Soorae, 2008). Results from an evaluation of 35 amphibian and 47 reptile translocation programs (for conservation, research, or to mitigate human-wildlife conflict) from 1991 to 2006 showed that $42 \%$ were successful, long-term success for $29 \%$ was still uncertain, and 28\% failed (Germano and Bishop, 2008). A review of 153 case studies of conservation translocation programs (most dating from 1984 onward) representing 168 animal species published in three volumes of the RSG Global Re-Introduction Perspectives (Soorae, 2008, 2010, 2011) showed different levels of success for different taxa (Schwartz, 2014). Programs for mammals (72\%) and fish $(68 \%)$ had the best success rates. Over half of reptile programs (56\%) and less than half of bird programs (42\%) were rated as successful. In contrast, a third of invertebrate and amphibian programs were rated as successful. Although these percentages seem to indicate improvement in program success rates, these case studies were submitted after an open request by the RSG and thus considered within an opportunistic dataset, rather than a random sample representing the whole dataset of conservation translocation programs. The success rate of these programs, nevertheless, indicates the need for improvement for all taxa.

Traditionally, in-situ and ex-situ practices have not been well integrated (Byers et al., 2013). In addition to responsible planning, data management for all levels of conservation programs including assessment, management and implementation require improvement. Evaluation of data at each level of the process is important and action can be altered if necessary for improved outcomes. Better records-keeping and data integration at all levels will facilitate sharing of information to provide better evidence of conservation success as well as evidence to support adaptive management processes. Sutherland et al. (2010) proposed standards for documenting all levels of reintroduction programs from initial planning, to methods for release, and post-release monitoring. The standards were recommended so that the collection of a comprehensive set of data could facilitate assessment for both successes and failure. In 2013, the updated RSG Reintroduction Guidelines contained a guideline for incorporating data management into conservation program design (IUCN/SSC, 2013) with recommendations for planning data management in advance so there would be no question on the data collection required for both ex-situ and in-situ components and the methods that should be used for monitoring the animals in the wild.

\section{INTEGRATION OF IN-SITU AND EX-SITU CONSERVATION PROGRAMS}

Zoos and aquariums, as conservation organizations, have long been involved in partnerships to work toward biodiversity conservation (Field and Dickie, 2007). Roughly one out of seven known threatened species on the planet can be found in a zoo or an aquarium (Conde et al., 2011) and captive breeding in such institutions may be the only practical conservation option left for some species whose habitats are dwindling. The authors showed that of 68 vertebrate species with improved conservation status as per their IUCN Red List designation, 13 species benefitted from conservation breeding with zoological institutions also contributing substantial logistical, technical or financial support for nine of those 13 species. Yet, 13 species out of 8143 listed in the threatened categories for vertebrate species on the Red List, (IUCN, 2016) is only a tiny proportion, illustrating the urgency to increase integrative conservation programs benefitting wild populations.

With the increasing need for collaboration between in-situ and ex-situ communities for overall conservation (Conway, 2007), the IUCN Conservation Planning Specialist Group 
(CPSG; previously Conservation Breeding Specialist GroupCBSG) proposed a "One Plan Approach" that considers all populations of a species both in the wild and in zoological facilities when planning conservation action when appropriate to do so (Byers et al., 2013). With a few exceptions, species conservation planning had traditionally been done in two parallel and separate tracks with in-situ specialists (field biologists, wildlife managers, and conservationists) monitoring and developing conservation action plans for threatened wild populations while zoo and aquarium specialists developed plans for sustaining ex-situ populations. With anthropogenic factors accelerating the extinction rate of species by 100 to a thousand times the natural rate (Pimm et al., 1995), it is imperative that species conservation strategies consider both in-situ and ex-situ communities when appropriate for holistic, integrative conservation planning and action.

Conservation action planning is dependent on the availability and accuracy of data on the threatened species to guide the formation of the plans to mitigate population declines. A Population and Habitat Viability Assessment (PHVA) (CBSG, 2017) is a workshop process combining face-to-face meeting of stakeholders with a Population Viability Analysis (PVA) component to predict extinction risk under current conditions and assess differing management strategies for conservation action. One of the most widely used PVA modeling tools is VORTEX (Lacy and Pollak, 2017), a computer program that analyzes the processes and factors affecting the viability of small populations. Required input data for VORTEX risk analysis includes biological (reproduction and genetic) as well as demographic parameters (birth, sex ratio, mating, dispersal, death). In addition, the frequency and severity of catastrophes (environmental, disease epidemics) and the impact of harvesting or supplementation (adding animals to the population) on population sizes are also important to consider. In considering the complexity of biological, physical and human systems that impact ecological systems, MetaModel Manager (Pollak and Lacy, 2013) was designed to provide an interface between Vortex, Outbreak (a modeling program that simulates infectious disease processes in wildlife populations; Lacy et al., 2012), and Spatial (a spatial data program that collects GIS data to track landscape changes due to climate change and/or human impacts; Pollak and Lacy, 2013). The PVA results for the use of Vortex or the more complex modeling process are heavily dependent on accessible data to limit uncertainty in the outcome of the analyses.

Incorporating sensitivity analysis (examination of the impacts of varied input parameters on projected population performance) in the PVA process can identify parameters or life history stages that will have the most effect on the trajectory of the population and thus inform management decisions (Mills and Lindberg, 2002). For many PVAs, data are unavailable for biological and demographic parameters if the species has not been adequately studied in the wild. In case studies of threatened species reintroduction or conservation translocation programs (Soorae, 2008, 2010, 2011) an analysis of 10 PHVAs and conservation workshops held between 1994 and 2004 (six for mammals, two for reptiles, one for birds and one for amphibians) showed that all the programs except one had insufficient data to adequately populate the fields necessary for PVA modeling. Eight noted that data for some demographic parameters were lacking, estimates were used based on best guesses, or data were from captive animals or work on similar species. The PHVAs and conservation workshops all noted the need for use of scientific-based evidence to manage and assess the programs and for genetic management of the captive populations (Schwartz, 2014).

Within a reintroduction or conservation translocation program, a monitoring plan for the released animals must be included in the management plan to assess the success of the conservation program (Nichols and Armstrong, 2012). Methods for intensive monitoring of individuals may include camera trapping, radio-telemetry, non-invasive genetic techniques, or capture-mark-recapture programs. Methods for less intensive monitoring may include survey counts, aerial surveys, or camera trap photos to determine presence. Acquiring long-term data on these post-release monitoring parameters is critical for analysis and adaptive management processes.

Finally, it is important to combine data from both ex-situ and in-situ components of the programs to identify potential trouble spots and assess the holistic conservation outcome. For example, the Western Pond Turtle Recovery Project was initiated in 1990 by the Woodland Park Zoo in collaboration with the Washington Department of Fish and Wildlife, the Oregon Zoo, and the U.S. Fish and Wildlife Service (Woodland Park Zoo, 2017) to re-establish this endangered species in Washington state. Populations of the western pond turtle (Actinemys marmorata) had drastically declined due to habitat fragmentation, disease and predation from non-native bullfrogs (Lithobates catesbeianus). Turtle eggs or hatchlings were collected from the wild and headstarted (hatched and/or reared at the zoo until large enough to prevent predation), and then were released back to wild habitats within their original range. The Western Pond Turtle PHVA (Pramuk et al., 2013) was conducted by (then) CBSG to evaluate the recovery program and results demonstrated the importance of the head-starting program to mitigate hatchling predation. An ulcerative shell disease was identified in 30\% of the released turtles as adults causing concern that survival and reproduction would be impacted. The cause of the disease was unknown but there was speculation that this could be linked to husbandry management while in the ex-situ institutions. The turtles' development may have been affected by inadequate nutrition or maintenance in habitats with poor water quality, thus making them more susceptible to ulcerative shell disease once they were released to the wild. The lack of adequate communication and standardization between the collaborating institutions as well as a lack of monitoring data on post-released hatchlings were of primary concern. The PHVA identified the data gaps and necessity for in-situ and ex-situ components of the program to work in concert with each other.

\section{INTEGRATION OF DATA MANAGEMENT PROCESSES}

Sophisticated records-keeping and population management tools have been developed for use in animal health and breeding management programs for ex-situ populations. Species 360 is a centralized database system that pools data on animal 
TABLE 1 | Core data for individual specimen records in ZIMS.

\begin{tabular}{|c|c|c|c|}
\hline Individual & $\begin{array}{l}\text { Reproduction/Breeding } \\
\text { Management }\end{array}$ & Behavior & Husbandry \\
\hline Taxonomy & Studbook ID & Social behavior & Enclosure requirements \\
\hline Birth/Hatch Date & Introduction date & Aggression & Diet \\
\hline Captive/Wild born & Copulation dates & Reproductive & Social grouping \\
\hline Parentage & Egg lay or Parturition date & Parturition & Reproduction \\
\hline Sex & Parturition behavior & Stereotypic & Incubation \\
\hline Rearing & Number of eggs or neonates & Interspecific & Rearing \\
\hline Birth Institution & Survival of neonates & Predatory & Training \\
\hline Collection location & Parental care & Feeding & Behavioral enrichment \\
\hline Holding institution & Contraception & Health & Human interaction \\
\hline \multirow[t]{2}{*}{ Terms of acquisition } & \multirow{2}{*}{$\begin{array}{l}\text { Pedigree: Parents } \\
\text { Siblings } \\
\text { Descendents }\end{array}$} & $\begin{array}{l}\text { Morphometrics } \\
\text { Development }\end{array}$ & Permits \\
\hline & & Body condition & CITES \\
\hline Individual identifiers & Enclosure/Environment & Parasite checks & \\
\hline Disposition date & Animals present & Injury & Exhibitor \\
\hline Receiving institution & Animal moves & Treatment & Somecenty \\
\hline Death date & Location in institution & Medications & \\
\hline \multirow[t]{6}{*}{ Media-photos, video } & Measurements & $\begin{array}{l}\text { Media-x-rays, photos, ultrasound } \\
\text { Necropsy }\end{array}$ & \\
\hline & Furniture & & \\
\hline & Life suppport system & & \\
\hline & Water quality & & \\
\hline & Temperature & & \\
\hline & Substrates & & \\
\hline
\end{tabular}

ZIMS, Zoological Information Management System.

populations in zoological facilities (Species360a, 2017 ${ }^{1}$ ). With a membership of over 1050 zoological institutions in 90 countries worldwide, Species360 offers the Zoological Information Management System (ZIMS), a global platform for managing animal records in real time (Species360b, 2017²). This global web-based database system manages life history, physiology, reproduction, behavior, and health data to facilitate cooperative animal husbandry and breeding management processes (Table 1). ZIMS' medical record functionality integrates health information with animal inventory and husbandry records within the animal's specimen record. In addition, ZIMS maintains institutional information to assign staff roles for access, a list of permits, transponder inventory, life support component inventory, and collection trip information (GPS location, equipment and vehicle types used, trip personnel, permits, species collected). Species360 membership is required (through yearly license fees) and member institutions/organizations enter data directly in the web-based ZIMS. Data are globally available in real time only to Species 360 members or the institution can designate a local view (not available to other institutions). In-situ partners would continue adding to each specimen's record after the individual's release, directly entering monitoring, health, reproduction, and mortality data into ZIMS.

\footnotetext{
${ }^{1}$ https://www.species360.org/ (Accessed May 4, 2017)

${ }^{2}$ ZIMS for Husbandry. Available online at: https://www.species360.org/productsservices/zims-for-husbandry (Accessed Sep 27, 2017).
}

Managing critical populations of endangered species in the wild has now become important as more conservation translocations of captive-bred or rescue/rehabilitated animals occur. Currently, only a few conservation translocation programs utilize these data management tools for holistic animal management (e.g., African penguin, Spheniscus demersus; Schwitzer et al., 2013; golden lion tamarin, Leontopithecus rosalia; Mickelberg and Ballou, 2013). There are few programs with a direct link between the ex-situ data management processes and databases used in monitoring and managing those animals released to the wild or for intensively managed wild populations (Schwartz, 2014). Species360 has added a new membership category for in-situ programs under Biological Institution-Reintroduction Facility/Site, facilitating integrated data management processes for reintroduction programs. The Rotoroa Island Field Site in New Zealand was one of the first Species360 members in this category. The Auckland Zoo in collaboration with the Rotoroa Island Trust and the New Zealand Department of Conservation developed a conservation action plan to create a new ecosystem on Rotoroa Island (Fraser et al., 2013) and introduce threatened invertebrates, fish, amphibians, reptiles, birds and mammals to Rotoroa Island over a 4-year period (2014 to 2017). Moko skink (Oligosoma mooch), brown kiwi (Apteryx mantelli), North Island saddleback (Philesturnus rufusater), whitehead (Mohoua albicilla), and South Island takahe (Porphyrio hochstetteri) have already been introduced, 
with transfers from Auckland Zoo to Rotoroa Island documented in ZIMS. Records for individuals released to the wild have documented history while in the zoological facility and extend to disposition data on release date and location. Use of ZIMS also establishes a link to monitoring data which is maintained separately in NZ Department of Conservation databases.

Zoos and aquariums are involved with in-situ conservation through many avenues including direct collaboration for returning animals to the wild (Dick and Gusset, 2010). As a global ZIMS, the Species360 central database offers the potential to pool data worldwide and contribute to an understanding of species biology, ecology, and conservation management. ZIMS offers an untapped capacity to link data management processes for animals that spend a part of their lives in zoological facilities and part in their natural environment as well as the potential for use for managed wild populations (Figure 1). Using ZIMS for long-term monitoring of populations in the wild could assist in identifying population parameters necessary for evaluation of conservation status (population size, growth, range, threat impact) and contribute to the IUCN Red List assessments.

\section{CONCLUSIONS}

To improve management and assessment of conservation programs, all available tools should be utilized and collaborations strengthened to reach the targets set by the Convention on Biological Diversity for species conservation. Data at each level of conservation action should be integrated within a holistic data management system to provide evidence necessary to assess the success of overall programs and to facilitate adaptive management. Use of ZIMS to link ex-situ and in-situ data management processes would:

- Facilitate a One Plan Approach for the integration of ex-situ and in-situ conservation action,

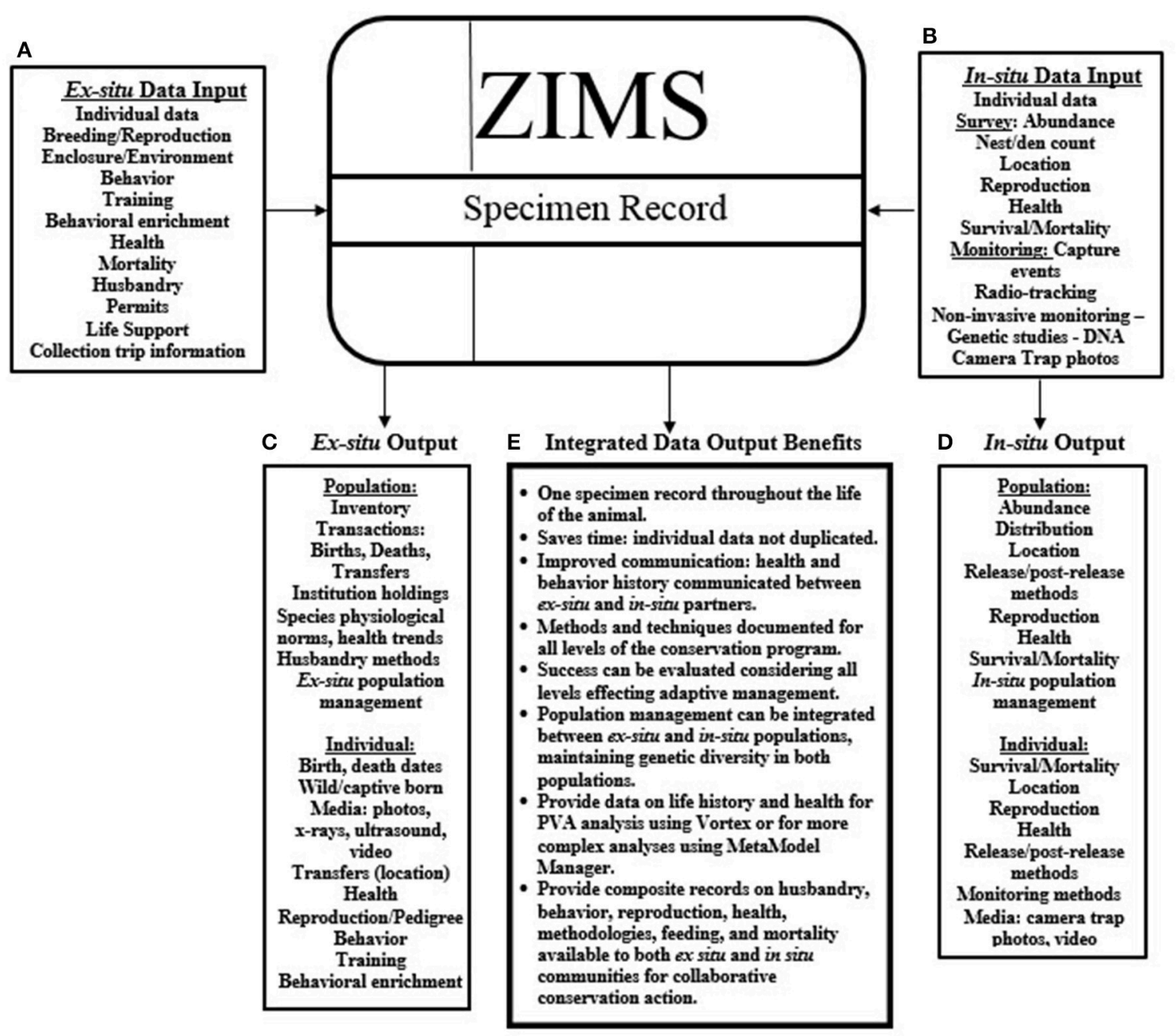

FIGURE 1 | Data management process for a single specimen record in a conservation program with ex-situ and in-situ components. (A) Data for ex-situ input into ZIMS. (B) In-situ data input either in a separate information system or as a continuance of ex-situ specimen record. (C) Output from ZIMS-ex-situ data alone. (D) Output from program databases-in-situ data alone. (E) Benefits of an ex-situ and in-situ integrated system. ZIMS, Zoological Information Management System. 
- Provide each individual animal with one record from the time of its birth to its death,

- Provide individual or group records on husbandry, behavior, reproduction, health, methodologies, feeding, training, transfers, and mortality that would be available to both the ex situ and in situ communities for collaborative conservation action,

- Facilitate genetic management of populations and metapopulations,

- Offer a database for pooled information to generate physiological normal values, identify life stage and reproductive attributes and act as a bio-surveillance mechanism for disease,

- Provide data on life history and health for PVA analysis using Vortex or for more complex analyses using MetaModel Manager.

Work has begun with Species360 to develop scientifically based recommendations for the use of ZIMS as a global database system that can provide a direct link between information collected on animals cared for in zoological facilities and on wild populations for conservation interventions (Schwartz, 2014). Going forward, Species360 needs to:

- Clarify Species360 membership roles for new in-situ conservation partners,

- Identify the role ZIMS can play with the current system,

\section{REFERENCES}

Beck, B. B., Rapaport, L. G., Stanley Price, M. R., and Wilson, A. C. (1994). "Reintroduction of captive-born animals," in Creative Conservation: Interactive Management of Wild and Captive Animals, eds P. J. S. Olney, G. M. Mac, and A. T. C. Feistner (London: Chapman and Hall), 265-286.

Bubb, P., Chenery, P. A., Herkenrath, P., Kapos, V., Mapendembe, A., StanwellSmith, D., et al. (2011). National indicators, monitoring and reporting for the Strategic Plan for Biodiversity 2011-2020. Cambridge: UNEP-WCMC. Available online at: https://www.bipindicators.net/system/resources/files/000/ 000/401/original/815.pdf?1480337853 (Accessed May 2, 2017).

Byers, O., Lees, C., Wilcken, J., and Schwitzer, C. (2013). The one plan approach: the philosophy and implementation of CBSG's approach to integrated species conservation planning. WAZA Magazine 14, 2-5. Available online at: http:// www.waza.org/en/site/conservation/integrated-species-conservation

Conde, D. A., Flesness, N. R., Colchero, F., Jones, O. R., and Scheuerlein, A. (2011). An emerging role of zoos to conserve biodiversity. Science 331, 1390-1391. doi: $10.1126 /$ science. 1200674

Conservation Breeding Specialist Group (CBSG). (2017). The PHVA Workshop Process. Apple Valley, MN. Available online at: http://www.cbsg.org/ourapproach/workshop-processes/phva-workshop-process (Accessed May 18, 2017).

Convention on Biological Diversity (2010). COP Decision X/2 The Strategic Plan for Biodiversity 2011-2020 and the Aichi Biodiversity Targets. Available online at: https://www.cbd.int/decision/cop/?id=12268 (Accessed May 18, 2017).

Conway, W. (2007). "Entering the 21st Century," in Zoos in the 21st Century, Catalysts for Conservation? eds A. Zimmermann, M. Hatchwell, L. Dickie, and C. West (Cambridge: Cambridge University Press), 12-21.

Dick, G., and Gusset, M. (eds.). (2010). Building a Future for Wildlife: Zoos and Aquariums Committed To Biodiversity Conservation. Gland: World Association of Zoos and Aquariums Executive Office.

Ewen, J. G., Armstrong, D. P., Parker, K. A., and Seddon, P. J. (eds.). (2012). Reintroduction Biology: Integrating Science and Management, 1 Edn. Hoboken, NJ: Wiley-Blackwell.
- Identify links that can be made between ZIMS and other biodiversity databases,

- Develop a module for data management for wild populations, including monitoring data through a collaboration of taxon specialists, Conservation Planning and Reintroduction Specialist Groups and Species360.

\section{AUTHOR CONTRIBUTIONS}

KS conducted the research, drafted and revised the manuscript; EP, TW, and LR contributed to research design, directed progress of the research, reviewed and gave final approval for the manuscript.

\section{ACKNOWLEDGMENTS}

This research was part of a dissertation submitted in partial fulfillment of the requirements for the degree of Doctor of Philosophy at George Mason University that was published at Mason and archived online (Schwartz, 2014). The research was supported by the Mason Departments of Environmental Science and Policy, and Biology, the Trevor Zoo in Millbrook, NY, and in-kind contributions from IUCN Conservation Planning Specialist Group and Species360. Jonathan D. Ballou served in a scientific advisory capacity, contributed to research design, directed progress of the research, and reviewed the manuscript.

Field, D. A., and Dickie, L. A. (2007). "Zoo coalitions for conservation," in Zoos in the 21st Century, Catalysts for Conservation? eds A. Zimmermann, M. Hatchwell, L. Dickie, and C. West (Cambridge: Cambridge University Press). 287-302.

Fischer, J., and Lindenmayer,. D. B. (2000). An assessment of the published results of animal relocations. Biol. Conserv. 96, 1-11. doi: 10.1016/S0006-3207(00)00048-3

Fraser, I., Wilcken, J., and Buley, K. (2013). Rotoroa Wildlife Management and Translocation Plan 2013-2038. Version 1.4. Auckland: Auckland Zoo.

Germano, J. M., and Bishop, P. J. (2008). Suitability of amphibians and reptiles for translocation. Conserv. Biol. 23, 7-15. doi: 10.1111/j.1523-1739.2008.01123.x

Gusset, M., Fa, J. E., Sutherland, W. J., and Horizon Scanners for Zoos and Aquariums. (2014). A horizon scan for species conservation by zoos and aquariums. Zoo Biol. 33, 375-380. doi: 10.1002/zoo.21153

IUCN/SSC (1998). Guidelines for Re-Introductions. Gland; Cambridge: IUCN/SSC Re-introduction Specialist Group.

IUCN/SSC (2013). Guidelines for Reintroductions and Other Conservation Translocations. Version 1.0. Gland: IUCN Species Survival Commission.

IUCN (2016). The IUCN Red List of Threatened Species. Version 2016-3. Available online at: http://www.iucnredlist.org (Accessed May 2, 2017).

Lacy, R. C., and Pollak, J. P. (2017). Vortex: A Stochastic Simulation of the Extinction Process. Version 10.2.7. Brookfield, IL: Chicago Zoological Society.

Lacy, R. C., Pollak, J. P., Miller, P. S., Hungerford, L., and Bright, P. (2012). Outbreak. Version 2.0. Apple Valley, MN: IUCN SSC Conservation Breeding Specialist Group.

Mickelberg, J., and Ballou, J. D. (2013). The Golden Lion Tamarin Conservation Programme's One Plan Approach. WAZA Magazine 14, 27-29.

Mills, L. S., and Lindberg, M. S. (2002). "Sensitivity analysis to evaluate the consequences of conservation actions," in Population Viability Analysis, eds S. R. Beissinger, and D. R. McCullough (Chicago, IL: University of Chicago Press), 338-366.

Nichols, J. D., and Armstrong, D. P. (2012). "Monitoring for reintroductions," in Reintroduction Biology, eds J. G. Ewen, D. P. Armstrong, K. A. Parker, and P. J. Seddon (Chichester; West Sussex: Wiley-Blackwell Publishing), 223-255. 
Pimm, S. L., Russell, G. J., Gittleman, J. L., and Brooks, T. M. (1995). The future of biodiversity. Science 269, 347-350.

Pollak, J. P., and Lacy, R. C. (2013). MetaModel Manager. Version 1.0. Brookfield, IL: Chicago Zoological Society.

Pramuk, J., Koontz, F., Tirhi, M., Zeigler, S., Schwartz, K., and Miller, P. (eds.). (2013). Western Pond Turtle (Actinemys marmorata) Population and Habitat Viability Assessment: Workshop Report (Apple Valley, MN: IUCN/SSC Conservation Breeding Specialist Group).

Pullin, A. S., Knight, T. M., Stone, D. A., and Charman, K. (2004). Do conservation managers use scientific evidence to support their decision-making? Biol. Conserv. 119, 245-252. doi: 10.1016/j.biocon.2003.11.007

Schwartz, K. R. (2014). Integration of In-Situ and Ex-Situ Data Management for Biodiversity Conservation via the ISIS Zoological Information Management System. Dissertation [Fairfax (VA)]: George Mason University, Department of Environmental Science and Policy. Available online at: http://hdl.handle.net/ $1920 / 9230$.

Schwitzer, C., Simpson, N., Roestorf, M., and Sherley, R. B. (2013). The African Penguin Chick Bolstering Project: A One Plan Approach to integrated species conservation. WAZA Magazine 14, 23-26.

Seddon, P. J., Armstrong, D. P., and Maloney, R. F. (2007). Developing the science of reintroduction biology. Conserv. Biol. 21, 303-312. doi: 10.1111/j.1523-1739.2006.00627.x

Soorae, P. S. (ed.). (2008). Global Re-Introduction Perspectives: Re-Introduction Case Studies from Around the Globe. Abu Dhabi: IUCN/SSC Re-introduction Specialist Group.
Soorae, P. S. (ed.). (2010). Global Re-Introduction Perspectives: Additional Case Studies from Around the Globe. Abu Dhabi: IUCN/SSC Re-introduction Specialist Group.

Soorae, P. S. (ed.). (2011). Global Re-Introduction Perspectives: More Case Studies from Around the Globe. Abu Dhabi: IUCN/SSC Re-introduction Specialist Group.

Sutherland, W. J., Armstrong, D., Butchart, S. H. M., Earnhardt, J. M., Ewen, J., Jamieson, I., et al. (2010). Standards for documenting and monitoring bird reintroduction projects. Conserv. Lett. 3, 229-235. doi: 10.1111/j.1755-263X.2010.00113.x

Wilson, E. O. (2016). Half-Earth. Our Planet's Fight For Life. New York, NY: Liveright Publishing Corporation.

Woodland Park Zoo (2017). Western Pond Turtle Recovery Project. Available online at: https://www.zoo.org/conservation/turtles (Accessed May 22, 2017).

Conflict of Interest Statement: The authors declare that the research was conducted in the absence of any commercial or financial relationships that could be construed as a potential conflict of interest.

Copyright (C) 2017 Schwartz, Parsons, Rockwood and Wood. This is an open-access article distributed under the terms of the Creative Commons Attribution License (CC $B Y)$. The use, distribution or reproduction in other forums is permitted, provided the original author(s) or licensor are credited and that the original publication in this journal is cited, in accordance with accepted academic practice. No use, distribution or reproduction is permitted which does not comply with these terms. 